\title{
Les principes pragmatiques de communication dans l'argumentation
}

Pragmatic Principles of Communication in Argumentation

\section{Scott Jacobs}

Translator. Marianne Doury

\section{OpenEdition \\ Journals}

Electronic version

URL: http://journals.openedition.org/aad/2078

DOI: 10.4000/aad.2078

ISSN: 1565-8961

Publisher

Université de Tel-Aviv

\section{Electronic reference}

Scott Jacobs, "Les principes pragmatiques de communication dans l'argumentation », Argumentation et Analyse du Discours [Online], 15 | 2015, Online since 15 October 2015, connection on 23 September 2019. URL : http://journals.openedition.org/aad/2078 ; DOI : 10.4000/aad.2078

This text was automatically generated on 23 September 2019.

\section{cc) (†)}

Argumentation \& analyse du discours est mis à disposition selon les termes de la licence Creative Commons Attribution - Pas d'Utilisation Commerciale - Pas de Modification 4.0 International. 


\title{
Les principes pragmatiques de communication dans l'argumentation
}

\author{
Pragmatic Principles of Communication in Argumentation
}

Scott Jacobs

Translation : Marianne Doury

1 Bon nombre de messages que les spécialistes en argumentation considèrent comme des paralogismes ont bien quelque chose de faux ou d'erroné, mais ne reposent pas pour autant sur des mensonges caractérisés, ou sur l'expression frontale et explicite de prémisses au contenu propositionnel inexact (cf. Coleman et Kay 1981). Et dans bien des cas, les destinataires peuvent être trompés, induits en erreur, roulés d'une façon ou d'une autre par des arnaques verbales et des escroqueries rendues possibles par l'expression d'implicatures conversationnelles ${ }^{1}$ - ou du moins, en amenant le destinataire à penser qu'il convient de tirer du message une implicature conversationnelle. Cet article cherche à élucider le fonctionnement de telles fraudes verbales. J'examinerai ces messages trompeurs afin de mettre en évidence l'importance des principes pragmatiques dans la compréhension des mécanismes argumentatifs, et de la façon dont ils peuvent être dévoyés.

2 Les implicatures conversationnelles sont, bien sûr, des phénomènes étroitement associés aux travaux de H. P. Grice (1989). Au cœur des réflexions de Grice se trouve l'idée que communiquer un message est une entreprise rationnelle. Lorsqu'un individu exprime ou interprète un message, il va au-delà de l'information que les règles du code confèrent à la syntaxe ou à la sémantique des phrases. Il dépasse ce niveau informationnel en construisant un contexte d'hypothèses et d'inférences qui font sens de ce qui a été dit, ainsi que de ce qui n'a pas été dit mais qui aurait pu être dit, de la façon dont ça a été dit, et du moment où ça a été dit. L'argumentation ne se réduit pas aux seuls mots ou aux phrases en elles-mêmes. Les mots et les phrases sont les indices à partir desquels le destinataire doit construire le sens du message. Le message est cet assemblage d'hypothèses et d'inférences qui s'ajoute à ce qui est exprimé, en comble les 
interstices, colore, et dans certains cas, remplace ce qui a été dit littéralement et directement (cf. Jacobs 2002). Il s'agit alors pour le destinataire de chercher à reconstruire un message qui fasse sens, et «faire sens » signifie, du moins pour Grice, " satisfaire autant que possible aux standards pour une action rationnelle».

3 Aussi, lorsque Grice a attiré l'attention sur les implicatures conversationnelles, cela concernait un point qui devrait éveiller l'intérêt des spécialistes en argumentation. Une implicature conversationnelle peut être vue comme

un mécanisme qui consiste à inférer ces attitudes, croyances et intentions implicites, qui font de ce qui est dit et fait, de la façon dont c'est dit et fait, en fonction du moment où c'est dit et fait, un moyen rationnel de faire passer un message.

Si cette caractérisation semble un peu alambiquée (comme son Principe de Coopération), les passages en italiques devraient permettre de rendre compte de l'idée de base. Pour les spécialistes d'argumentation, Grice montre que la façon dont les gens raisonnent pour construire des représentations des argumentations est soumise à des normes rationnelles, tout autant que la façon dont les gens raisonnent à partir de, et avec, des représentations des argumentations ${ }^{2}$. Les procédures par lesquelles les locuteurs construisent et offrent leurs argumentations aux destinataires, et les procédures par lesquelles ces destinataires accèdent à ces argumentations (au contenu des prémisses et des conclusions, à la configuration de l'inférence qui les lie, et à leur force) sont susceptibles de faire l'objet d'une évaluation normative, basée sur des standards rationnels, au même titre que les argumentations elles-mêmes.

5 J'attache une importance relative à la formulation qu'il conviendrait de choisir pour les standards de communication rationnels. Le principe de coopération et les maximes conversationnelles de Grice ont le mérite de distinguer les attentes de véracité, d'informativité, de pertinence et de clarté, chacun de ces critères étant mis en relation avec les objectifs de la communication ${ }^{3}$. Appliqués à l'objectif de justifier une proposition, ils se rapprochent beaucoup, par exemple, des standards établis par Johnson et Blair (2006) relatifs à l'acceptabilité, la suffisance et la pertinence des prémisses. Et les spécialistes d'argumentation reconnaitront dans l'hypothèse que le locuteur respecte le Principe de coopération et les maximes conversationnelles - ou du moins, tente de les respecter autant qu'il le peut - quelque chose comme le principe de charité, qui guide les reconstructions analytiques des argumentations (Gauker 1986, Wilson 1959). Ainsi, la théorie gricéenne de l'implicature conversationnelle peut être vue comme situant les standards normatifs de la conduite rationnelle au cœur de l'acte même de communication.

6 La présomption de Pertinence Optimale de Sperber et Wilson (1995) met l'accent sur l'attente d'efficacité dans la communication (qui constitue le gros lot auquel aspirent tous les communicants), attente que Grice ne mentionne pas ${ }^{4}$. Le principe $\mathrm{R}$ et le principe Q de Horn (1984) mettent au jour dans les maximes de Grice quelque chose comme le Principe du moindre effort de Zipf (1949) : chacun doit faire ce qu'il a à faire, mais pas plus ${ }^{5}$. Les principes Q, M et I de Levinson (2000) attirent l'attention sur le rôle central que jouent, dans la production et l'interprétation des inférences, les savoirs relevant du sens commun et les moyens (a)normaux d'expression ${ }^{6}$.

7 Tous ces standards peuvent être appliqués de façon plus spécifique. Dans les exemples qui suivent, j'en proposerai des formulations en termes de "Formes normales ", « àpropos informationnel " et "suffisance informationnelle». Ces formulations ne sont sans doute rien de plus qu'une autre façon d'exprimer ce que d'autres ont déjà dit, mais 
elles me semblent utilement démêler certaines des implications de ces autres standards.

8 L'idée que je souhaite explorer en relation à ces trucages, escroqueries ou fraudes verbales est la suivante : les principes qui rendent la communication possible peuvent aussi être les principaux ressorts de duperies verbales. Ces standards rationnels de la communication fonctionnent comme des présomptions, comme des principes de charité, si vous voulez. C'est la présomption qu'ils sont satisfaits qui sous-tend les hypothèses que l'on fait et les implications que l'on tire, et que l'on appelle des «implicatures conversationnelles $»^{7}$. Les tromperies et arnaques exploitent ces présomptions de façon pernicieuse, dans la mesure où elles peuvent amener le destinataire à en inférer des informations fausses, à en tirer des conclusions injustifiées; elles peuvent aussi simplement permettre à des messages frauduleux de passer inaperçus.

\section{Cas 1: Discours du Sénateur Edward Kennedy sur l'accident de Chappaquiddick}

9 Afin d'éclairer ce que je viens de dire, considérons le cas de l'intervention télévisée du sénateur Edward Kennedy, diffusée en 1969 dans tout le pays, au sujet des événements survenus sur l'île de Chappaquiddick, au cours de la nuit où, après une fête dans une résidence d'été, sa voiture semble avoir quitté la route et être tombée d'un pont ${ }^{8}$. Sa passagère, Mary Jo Kopechne, s'est noyée; pourtant, à aucun moment au cours de la nuit, le sénateur n'a signalé l'accident. Il a regagné sa chambre d'hôtel, et a enfin rapporté l'accident le matin suivant, alors que la police avait déjà découvert la voiture immergée, et le corps de Mademoiselle Kopechne à l'intérieur du véhicule. Le discours du sénateur est intervenu alors qu'une semaine entière s'était écoulée après l'annonce de l'accident, semaine au cours de laquelle les médias se sont livrés à d'intenses spéculations sur le comportement suspect du Sénateur Kennedy. Parmi les spéculations en circulation, on trouve l'hypothèse que le sénateur avait tenté de dissimuler l'accident, et avait tout simplement laissé Mademoiselle Kopechne se noyer.

On reproduira ci-dessous deux extraits tirés de ce discours (Kennedy 1969). Le premier rapporte les événements qui ont suivi immédiatement l'accident, après l'échec des tentatives du sénateur pour plonger et atteindre la voiture immergée afin de retrouver Miss Kopechne et de la tirer du véhicule. Le second extrait indique ce que le sénateur a fait à son réveil dans sa chambre d'hôtel le matin suivant.

Extrait 1

Après être resté étendu dans l'herbe, épuisé, pour une durée indéterminée, je n'ai pas cherché directement un téléphone mais je suis revenu à pied à la résidence où la fête avait lieu, et j'ai demandé de l'aide à deux amis, mon cousin, Joseph Gargan, et Paul Markham, et je les ai conduits immédiatement sur le lieu des faits - c'était un peu après minuit - afin de tenter à nouveau de plonger et localiser Miss Kopechne.

Extrait 2

Dans la matinée, j'ai retrouvé un peu de lucidité et j'ai cherché à joindre un conseiller juridique de ma famille, Burk Marshall, à partir d'un téléphone public situé à la gare du ferry-boat côté Chappaquiddick ; c'est alors que j'ai tardivement signalé l'accident à la police de Martha's Vineyard. 
11 Le discours, dans son ensemble, constitue un exercice d'apologie politique (Ware et Linkugel 1973). Il s'agit d'un discours d'auto-défense, et les détails de l'histoire qui y figurent implicitent [anglais implicate] des éléments d'information pertinents au regard d'une ligne argumentative qui, si elle était assumée explicitement, conduirait à la conclusion que le sénateur n'a rien fait qui soit répréhensible au cours des événements qui ont précédé ou suivi la mort de Miss Kopechne (Jacobs, 1993).

Ces deux extraits contribuent à présenter des informations qui appuient cette version. Ils mettent en scène un jeune homme plein de bonne volonté mais égaré et désorienté, cherchant aide, soutien, conseil auprès de ses amis et de sa famille. Le sénateur n'affirme pas explicitement que ses actions n'ont pas été motivées par la volonté de dissimuler son implication dans l'accident. Rien, dans ce qu'il dit, ne mentionne même un tel scénario. Mais l'impression générale qui se dégage de son récit des faits est clairement incompatible avec une telle lecture des événements, et il ne fait aucun doute que ces deux extraits visent à soutenir cette argumentation.

On verra que c'est bien ce qui est au cœur du message en examinant les expressions suivantes: "deux amis, mon cousin, Joseph Gargan, et Paul Markham », "un conseiller juridique de la famille, Burk Marshall ». Ces deux caractérisations sont exactes, et toutes deux sont incontestablement pertinentes pour expliquer la conduite de Kennedy. Mais la vérité et la pertinence des descriptions considérées en elles-mêmes sont secondaires au regard de ce qu'évoquent ces désignations dans le savoir commun.

14 Harvey Sacks (1972) considère de telles désignations comme des "procédés de catégorisation par appartenance ». Ces termes activent des schémas interprétatifs ; selon Sacks, des désignations comme « ami », « cousin » ou " conseiller juridique de la famille » confèrent une signification particulière et assignent des intentions spécifiques à des activités comme, dans le cas qui nous concerne, « chercher de l'aide » ou « passer un appel». En termes pragmatiques, recourir à ces désignations génère tout un ensemble d'implicatures faibles sur les relations sociales, les motivations qui soustendent l'action, les lignes de raisonnement et les plans d'action, reposant sur des typifications de "ce que chacun sait» (Cicourel 1970). Autrement dit, l'emploi et l'interprétation de telles désignations supposent que l'on admette quelque chose comme le principe suivant :

Formes normales

Ce qui est dit doit être pris dans sa signification ordinaire, constitue un emploi ordinaire dans des circonstances ordinaires, sauf indication contraire.

Par le recours à des termes comme " ami » ou « famille », on considérera que Kennedy entend mettre en lumière des propriétés typiques de tels individus ou relations ${ }^{9}$.

16 Et l'emploi de ces désignations présuppose aussi qu'elles sont plus adéquates que d'autres désignations qui auraient été possibles. Autrement dit, en interprétant ces extraits, les téléspectateurs activent le principe interprétatif général qui suit:

À-propos informationnel ${ }^{10}$ :

Les désignations utilisées sont les plus justes par rapport aux autres descriptions disponibles: elles activent le plus grand nombre d'implicatures exactes et pertinentes, et le plus petit nombre d'implicatures erronées ou non-pertinentes.

Il faut poser ce principe (en association avec celui des Formes normales) pour faire la lumière sur l'impact argumentatif attaché à ces désignations. On peut mettre en évidence le rôle de cette présomption pragmatique d'à-propos informationnel en considérant les descriptions alternatives qui auraient été possibles. Qu'en serait-il par 
exemple si, au lieu des désignations relevées plus haut, Kennedy avait utilisé la caractérisation suivante :

\section{Extrait 1}

Après être resté étendu dans l'herbe, épuisé, pour une durée indéterminée, je n'ai pas cherché directement un téléphone mais je suis revenu à pied à la résidence où la fête avait lieu, et j'ai demandé de l'aide à deux avocats, Joseph Gargan, qui est depuis des années mon conseiller politique, et Paul Markham, l'ancien procureur général de l'Etat du Massachussetts, et je les ai conduits immédiatement sur le lieu des faits c'était un peu après minuit - afin de tenter à nouveau de plonger et localiser Miss Kopechne.

Extrait 2

Dans la matinée, j'ai retrouvé un peu de lucidité et j'ai cherché à joindre celui qui, depuis des années, conseille la machine politique des Kennedy, et un homme que Bobby Kennedy considérait comme le juriste le plus pointu qu'il ait jamais rencontré, ancien Procureur général assistant, Burk Marshall, à partir d'un téléphone public situé à la gare du ferry-boat côté Chappaquidick; c'est alors que j'ai tardivement signalé l'accident à la police de Martha's Vineyard.

Ces descriptions sont également véridiques. Et on peut se demander si elles ne sont pas également pertinentes pour expliquer la conduite de Kennedy. Mais l'emploi de ces descriptions implicite des motivations et des activités bien différentes, et ne communique aucunement le sentiment que Kennedy ne cherchait pas à dissimuler son implication dans les événements de la nuit, ou qu'il était incapable de concocter une version susceptible de mettre sa carrière politique à l'abri d'un scandale qui lui aurait été fatal. Ces formulations vous paraissent difficilement comparables avec ce que Kennedy a effectivement dit? Substituons-leur les désignations suivantes, plus concises, et approximativement de même longueur que les expressions originales :

Extrait 1

Après être resté étendu dans l'herbe, épuisé, pour une durée indéterminée, je n'ai pas cherché directement un téléphone mais je suis revenu à pied à la résidence où la fête avait lieu, et j'ai demandé de l'aide à deux avocats, mon assistant, Joseph Gargan, et Paul Markham, et je les ai conduits immédiatement sur le lieu des faits c'était un peu après minuit - afin de tenter à nouveau de plonger et localiser Miss Kopechne.

Extrait 2

Dans la matinée, j'ai retrouvé un peu de lucidité et j'ai cherché à joindre un ancien Procureur général assistant, Burk Marshall, à partir d'un téléphone public situé à la gare du ferry-boat côté Chappaquidick; c'est alors que j'ai tardivement signalé l'accident à la police de Martha's Vineyard.

$19 \mathrm{Ou}$ encore, plutôt que "un ancien Procureur général assistant", présentons simplement Burk Marshall comme "un conseiller politique des Kennedy». De nombreuses désignations exactes (et potentiellement pertinentes) peuvent être utilisées pour référer à Gargan, Markham et Marshall, et pour contextualiser plus spécifiquement la signification de ce que Kennedy faisait lorsqu'il a "demandé de l'aide » à ces hommes ou « a cherché à joindre » l'un d'entre eux.

Ce que je veux à présent souligner ici, ce sont les réactions de surprise suscitées par la lecture de ces descriptions alternatives - partant de l'hypothèse qu'elles sont également vraies. On peut se demander si, au-delà de leur véracité, elles sont révélatrices des qualités que Kennedy avait à l'esprit en s'adressant à ces personnes, et si elles pourraient elles aussi être pertinentes pour expliquer la conduite de Kennedy. Le sentiment de perplexité et de malaise qu'elles suscitent témoigne de l'existence d'un 
principe dont la satisfaction était présupposée, et qui est maintenant mise en doute. Je suggère que ce principe revient à ce que j'ai appelé plus haut l'à-propos informationnel.

21 J'ai invité le lecteur à comparer ce qui a été dit à ce qui aurait pu être dit ; adoptons à présent la démarche qui consiste à mettre en regard ce qui a été dit et ce qui a été passé sous silence. On voit à nouveau que les destinataires d'un message s'appuient sur une sorte de présomption pragmatique lorsqu'ils interprètent un discours. Selon cette présomption, si une information vraie n'a pas été mentionnée, c'est qu'elle n'avait pas d'importance; elle n'était pas requise pour atteindre les objectifs communicationnels poursuivis. Ainsi, par défaut, on considère que ce qui a été mentionné est informationnellement suffisant pour les fins du message.

Suffisance informationnelle

Ce qui est vrai, mais passé sous silence, et qui ne peut être aisément inféré, ne doit pas être pertinent au regard des objectifs de l'échange (ou n'est sans doute pas considéré par le locuteur comme vrai, pertinent et/ou difficile à inférer). Ce qui est mentionné doit être informationnellement suffisant pour les besoins de l'échange.

Considérons à présent le récit alternatif suivant, comportant cette fois une information additionnelle exacte mais préalablement omise. Qu'en serait-il si Kennedy avait ajouté à l'extrait 1 le passage qui suit, que je mets en italiques :

\section{Extrait 1}

Après être resté étendu dans l'herbe, épuisé, pour une durée indéterminée, je n'ai pas cherché directement un téléphone mais je suis revenu à pied à la résidence où la fête avait lieu, et j'ai demandé de l'aide à deux amis, mon cousin, Joseph Gargan, et Paul Markham, et je les ai conduits immédiatement sur le lieu des faits - c'était un peu après minuit - afin de tenter à nouveau de plonger et localiser Miss Kopechne. Je n'ai alerté aucune des cinq femmes ou aucun des trois autres hommes qui participaient à la fête, parmi lesquels, Raymond LaRosa, un pompier entraîné au sauvetage en plongée.

L'information concernant ceux qui n'ont pas été alertés semble avoir un impact argumentatif évident dès lors qu'elle est portée à la connaissance du destinataire. Il me semble que bon nombre d'entre nous considéreraient cette omission comme frauduleuse s'il s'avérait que Kennedy avait eu connaissance de l'entraînement de Raymond LaRosa ${ }^{11}$. Au minimum, la nouvelle version de l'extrait 1 proposée ci-dessus suggère que le discours de Kennedy ressemble davantage à une argumentation unilatérale d'auto-justification qu'à la simple description de "ce qui s'est passé" annoncée par Kennedy ${ }^{12}$. Ne pas communiquer à l'auditoire une telle information semble favoriser les inférences trompeuses que l'on désigne habituellement comme des paralogismes. Et le fait que nous soyons dès lors assaillis par ces intuitions, doutes et soupçons indique que l'on s'attend à ce que soit respecté un principe de Suffisance informationnelle.

\section{Cas 2. La carte Willie Horton}

Plus encore: j'avancerai que bien des paralogismes réussissent en jouant sur les présomptions générales de l'auditoire, qui tient ce que le discours véhicule pour vrai, justifié, informatif, et d'une utilité adaptée aux objectifs apparents de l'échange. Prenons la question de l'ambiguïté. Les théoriciens de l'argumentation considèrent dans l'ensemble que l'ambiguïté pose problème quant à la précision des inférences. Quand des termes sont vagues, ambigus ou polysémiques, il est impossible d'en tirer 
des inférences fiables et précises ${ }^{13}$. Mais l'ambiguïté peut être plus qu'un simple problème logique. D'ordinaire, les destinataires résolvent les ambiguïtés potentielles, ou ne les remarquent même pas. Les paralogismes d'ambiguïté peuvent avoir une dimension pragmatique qui exploite la façon dont plusieurs interprétations peuvent se faire jour. L'une de ces interprétations est relativement inoffensive : elle est exacte, mais n'est guère pertinente argumentativement, et sa force est limitée. L'autre interprétation aurait une pertinence argumentative bien supérieure, sous réserve qu'elle soit vraie. Mais c'est là que le bât blesse : l'auditoire présume la vérité de ce qui lui est dit, et sélectionne l'interprétation qui semble la plus pertinente au regard des intentions manifestes de celui qui argumente. Et dans des cas de ce genre, le contenu de cette interprétation est en fait erroné, et le destinataire est induit en erreur. élections présidentielles de 1988 :

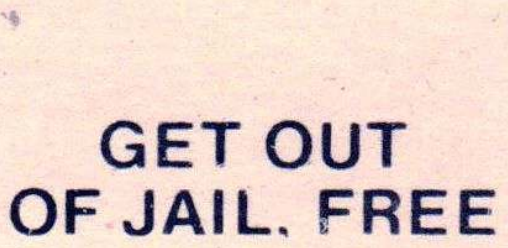

OF JAIL. FREE

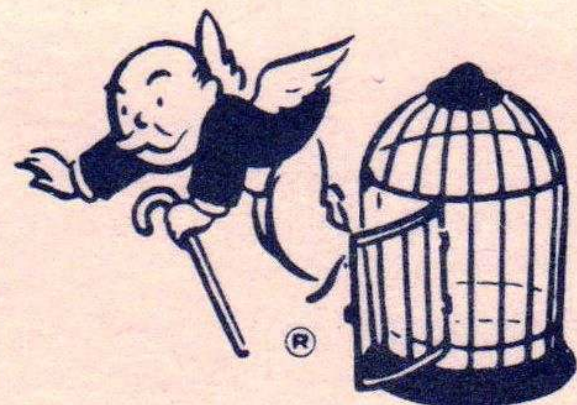

COMPLIMENTS OF MICHAEL DUKAKIS

DISTRIBUTED BY COLLEGE REPUBLICAN NATIONAL COMMITTEE 1988 CRNC

Get Out of Jail, Free. Compliments of Michael Dukakis

Carte du collège électoral du Comité National Républicain, 1988 (recto) 
Michael Dukakis's furlough plan allowed convicted 4 murderers to take a weekend leave from prison. One, Willie Horton left and never came back. Instead he viciously raped and beat a woman while her fiancé was forced to helplessly listen to her screams.

This is only one example of many. In the last several years, Mike Dukakis has furloughed more than one murderer per day.

Mike Dukakis is the killer's best friend, and the decent, honest citizens' worst enemy.

Get Out of Jail, Free. Compliments of Michael Dukakis

Carte du collège électoral du Comité National Républicain, 1988 (verso)

Rappelons que le plateau de jeu du Monopoly comporte une case « Allez en Prison». Les joueurs qui s'arrêtent sur cette case ne peuvent pas jouer durant trois tours, à moins de payer une pénalité qui leur permet de sortir immédiatement. S'il est en possession de la carte "Vous êtes libéré de prison », le joueur peut sortir instantanément de prison sans payer la moindre pénalité et recommencer à jouer. Au dos de la carte distribuée par l'équipe de campagne de Bush Senior, on peut lire la désormais célèbre histoire de Willy Horton :

Le plan de Michael Dukakis relatif à la liberté conditionnelle a autorisé les prisonniers reconnus coupables de meurtre à sortir de prison pour un week-end. L'un d'entre eux, Willie Horton, est sorti et n'est jamais revenu. Il a préféré sauvagement violer et battre une femme, devant son fiancé réduit à écouter ses cris, impuissant.

Ce n'est qu'un exemple parmi tant d'autres. Au cours des dernières années, Mike Dukakis a autorisé la sortie de prison de plus d'un meurtrier par jour. Mike Dukakis est le meilleur ami des assassins, et le pire ennemi des honnêtes citoyens.

Willie Horton était un prisonnier noir, condamné à la prison à vie pour un meurtre épouvantable (d'un homme blanc). Il a été autorisé à sortir par l'état du Massachussetts sous la mandature de Michael Dukakis en tant que gouverneur (Simon, 1990). Ainsi que le suggère la carte, Willie Horton a été « libéré de prison ».

L'ambiguïté de la carte réside toute entière dans la formulation "Ce n'est qu'un exemple parmi tant d'autres ». "Parmi tant d'autres quoi ?", pourrait-on demander. Est-ce seulement un exemple parmi tant d'autres exemples de prisonniers condamnés pour meurtre qui ont été autorisés à sortir pour le week-end ? Ou est-ce seulement un exemple parmi tant d'autres exemples de prisonniers condamnés pour meurtre qui, lors de leur week-end de permission, ont commis un crime haineux, comme sauvagement violer et battre une femme alors que son fiancé impuissant était réduit à l'entendre hurler ${ }^{14}$ ? La première interprétation, au mieux, n'étaye que faiblement l'objectif présumé de la carte : montrer que Michael Dukakis n'est pas celui qu'il faut 
pour assumer la fonction de Président des Etats-Unis d'Amérique et qu'il ne convient donc pas de voter pour lui.

Selon cette première interprétation, l'histoire racontée au dos de la carte peut être vue comme reflétant une erreur de jugement troublante. Mais lui accoler le commentaire "Ce n'est qu'un exemple parmi tant d'autres " pourrait sembler en réalité en atténuer l'impact. L'information est formulée de façon si prudente qu'elle en devient non pertinente, ou même contre-productive. Après tout, s'il y a eu «tant d'autres " permissions accordées, et seulement un cas comme celui qui est rapporté, alors les agissements de Willie Horton pourraient être vus comme une exception aberrante à la règle.

La seconde interprétation, à l'inverse, amplifie de bien des manières un des pires cauchemars de la classe moyenne blanche aux Etats-Unis (la femme et son fiancé étaient tous deux blancs). Cette interprétation constitue potentiellement une raison décisive aux yeux de bon nombre d'électeurs pour conclure que Michael Dukakis n'est pas à même d'assumer la fonction de Président des Etats-Unis d'Amérique (c'est-à-dire de «Protecteur-en-chef»). Et pour en arriver à cette dernière interprétation du message, il suffit de présumer que le collège électoral du Comité National Républicain produit l'information qu'il estime pertinente et suffisante pour accomplir son objectif manifeste, qui est de soutenir la candidature de George H. W. Bush. Quoi qu'il en soit, cette interprétation plus radicale comporterait une information erronée. Il n'existe aucun autre individu condamné pour meurtre qui, ayant bénéficié d'une telle permission, se soit rendu coupable d'agissements similaires à ceux commis par Willie Horton. Mais, bien entendu, le comité de campagne de Bush fait l'hypothèse que les électeurs, présumés mal informés, n'en sauront rien. Et ils exploitent l'existence de l'interprétation plus modeste, voire insignifiante, qu'ils pourront toujours mettre en avant si la version radicale venait à être contestée. Ils tiennent ainsi en réserve une stratégie de dénégation plausible. Ils peuvent toujours, en cas de besoin, prétendre qu'ils voulaient juste dire que Michael Dukakis avait validé un programme faisant bénéficier de nombreux prisonniers d'une permission; et qu'une telle mesure n'était pas bonne ; et le paralogisme potentiel est ainsi dissout par une interprétation légitime mais insignifiante.

31 Il faut encore noter que la carte contrevient également au principe de la Forme normale. L'expression «Le plan de Dukakis sur les permissions » suppose normalement que ce programme a été planifié et lancé par Dukakis. C'est l'état de fait typique, normal, auquel devraient renvoyer l'utilisation du complément du nom "de Dukakis », ainsi que le terme "plan» (par opposition à " programme»). Il est vrai que, après la tragédie d'Horton, Dukakis s'est opposé à la révocation du programme accordant des permissions aux prisonniers condamnés à la prison à vie. Mais la loi instituant ce programme avait été en fait conçue et votée par le précédent gouverneur, un Républicain, qui s'était lui-même inspiré d'un programme introduit par le gouverneur de Californie Ronald Reagan (l'homme sous la présidence duquel George Bush assumait la fonction de vice-président). On voit ici clairement en quoi la carte transgresse soit le principe de la Forme normale, soit le principe de la Suffisance informationnelle, soit peut-être même les deux. 


\section{Cas 3 : Le mémo de « 60 minutes »}

Les paralogismes qui exploitent les présomptions de pertinence n'implicitent pas toujours un contenu informationnel inexact. Ils n'ont pas besoin non plus de s'appuyer sur des informations invérifiables pour «marcher». Ils se produisent parfois "à découvert ", comme dans l'exemple qui suit. Il s'agit d'un mémo, imprimé sur un papier à en-tête du Bureau du Président de l'Université d'Arizona.

Brève réponse à 60 minutes

L'émission 60 minutes consacrée aux universités en général, et à l'Université d'Arizona en particulier, nous offre une bonne opportunité: elle nous donne l'occasion de parler de l'enseignement supérieur. Voici des réponses à quelques questions ardues ou des réactions à des commentaires qui ont été soulevés à plusieurs reprises.

Q.60 Minutes a dit que les parents paient 15000 dollars de frais de scolarité afin de financer la recherche menée par les professeurs. Est-ce vrai ?

R. C'est faux. L'émission a confondu l'Université d'Arizona et la situation de quelques petites universités privées. Les frais de scolarité pour l'université d'Etat sont de 1800 dollars, et non de 15000 dollars. De plus, les sommes versées comme frais de scolarité ne sont jamais utilisées pour soutenir la recherche, point à la ligne. C'est même l'inverse qui est vrai, parce que $80 \%$ de l'équipement utilisé par les étudiants en science a été acquis sur les financements de recherche.

Q. Est-il exact que $87 \%$ des étudiants en première année ont des assistants comme enseignants?

R. C'est faux. 60 Minutes a mal interprété les statistiques. Si un professeur en titre dispense un enseignement de trois heures hebdomadaires devant un auditoire de 200 étudiants, ça compte pour un seul cours. S'il répartit ses étudiants en 9 groupes de cours d'une heure en laboratoire, et que chacun de ces cours est pris en charge par un assistant, alors chaque groupe compte comme un cours. C'est comme ça que 60 Minutes a conclu que $90 \%$ des étudiants avaient des assistants comme enseignants. La réalité, c'est que du point de vue d'un étudiant, la part d'enseignement assurée par les assistants n'est que de $25 \%$.

Q. Est-il exact que dans le département d'Anglais, aucun professeur en titre n'enseigne l'expression écrite aux étudiants de première année?

R. Quarante membres du corps enseignant donnent des cours d'expression écrite aux premières années. Certains d'entre eux ont enseigné ailleurs, et sont revenus à l'Université d'Arizona pour suivre des cours plus avancés; certains sont enseignants à temps partiel dans notre université. Ce qui compte, c'est que, indépendamment de qui en enseigne les techniques de base, notre programme d'expression écrite est considéré comme un modèle du genre. Juste une semaine avant l'émission 60 Minutes est paru un article dans les Chronicles for Higher Education, qui citait des pans entiers de notre programme comme modèle d'articulation de l'enseignement et de la recherche dans les classes.

Ce document a été distribué chez les marchands de presse de Tucson et dans les départements du campus au milieu des années 1990. Il constitue une réponse à un reportage sur l'enseignement supérieur aux Etats-Unis diffusé dans l'émission télévisée 60 Minutes. Lors de cette émission, les formations dispensées à l'Université d'Arizona avaient été la cible de mises en cause sérieuses, et la note visait à réfuter nombre d'allégations qui avaient été avancées, lors de l'émission, sur ce qui se passait dans cette université.

La réponse à la troisième question (" est-il exact que dans le département d'Anglais, aucun professeur en titre n'enseigne l'expression écrite aux étudiants de première année?») est particulièrement intéressante. Elle illustre un type de tromperie 
différent du précédent, qui consistait à encourager une implicature inexacte. A première vue, on peut penser que cette contribution constitue une réponse indirecte, à peine plus compliquée que dans l'échange «Avez-vous du feu? - «Je ne fume pas », où «Je ne fume pas" doit être compris comme implicitant "Non». En réalité, la réponse n'en est pas vraiment une, même si, superficiellement, on pourrait penser le contraire. Il s'agit plutôt d'un cas de ce que Leah Polcar et moi considérons comme une « dérobade » (Polcar et Jacobs 1998). Ça ressemble à une réponse - indirecte, certes, mais réponse quand même ; mais il ne s'agit en fait pas d'une réponse, même indirecte. Une intervention réactive est formulée de telle sorte qu'elle puisse passer pour une réponse indirecte ; mais si on cherche à préciser en quoi consiste cette réponse, on se trouve en difficulté ; elle déjoue toute tentative de spécification et se dérobe à l'examen (ici, en changeant de sujet). Et seule une lecture attentive permet de voir ce qui est à l'œuvre ici.

Un lecteur attentif remarquerait que, contrairement aux réponses aux deux premières questions, la réaction à la troisième question ne commence par aucune dénégation claire ; n'y figure aucun « C'est faux ».

De plus, les catégories mobilisées dans la réponse ne correspondent pas à la catégorie présente dans la question. La question portait sur la présence ou non de "professeurs » assurant les cours d'expression écrite aux premières années. Je présume que la plupart des lecteurs américains comprendraient le terme de "professeur » comme référant aux membres ordinaires, prototypiques, de cette catégorie - des membres du personnel enseignant titulaires en exercice, c'est-à-dire des professeurs en poste, des professeurs associés ou des assistants.

Mais notons que la réponse mentionne quarante «membres du corps enseignant » qui assurent les cours d'expression écrite aux étudiants de première année. De qui s'agit-il exactement? On nous indique que "certains d'entre eux " «ont enseigné ailleurs, et sont revenus à l'Université d'Arizona pour suivre des cours plus avancés ». Il s'agit sans doute d'étudiants de troisième cycle, dans la mesure où les professeurs ne suivent pas de cours, mais en donnent. Ils correspondraient à la catégorie des "enseignants assistants ", catégorie qui figure au côté des "professeurs titulaires » mentionnés en réponse à la seconde question, et qui est absente de cette réponse.

On nous dit aussi que « certains sont des enseignants à temps partiel ». Les membres de cette catégorie de personnel sont habituellement appelés « lecteurs», ou " professeurs auxiliaires ", mais ne sont pas non plus des professeurs au sens habituel du terme.

Mais on ne nous dit jamais clairement si des professeurs, au sens habituel du terme, figurent parmi ce qui reste des quarante « membres du corps enseignant » assurant des cours d'expression écrite. La soi-disant réponse se contente de déplacer la discussion sur un autre thème (« ce qui est important, c'est que... »).

Et plus on s'y attarde, moins il est possible de décider si le document cherche à impliciter une réponse quelconque. Les deux premières phrases de cette "réponse " constituent une sorte d'aveu indirect que certains de ceux qui enseignent l'expression écrite aux étudiants de première année ne sont pas des professeurs. Mais un lecteur moins scrupuleux pourrait bien être amené à penser, à tort, que les enseignants mentionnés dans la note sont, en effet, des professeurs. Un lecteur attentif pourrait inférer, par une sorte de calcul pratique, que les fluctuations dans le choix des désignations traduisent le fait qu'aucun professeur n'enseigne l'expression écrite aux étudiants de première année. Mais la formulation en elle-même ne va pas jusqu'à 
admettre cela. Et, cette fois encore, la note ne semble pas impliciter non plus que, si certains, parmi ceux qui enseignent, ne sont pas professeurs, d'autres le sont. Il se trouve qu'en réalité, à ce moment-là, aucun professeur n'enseignait l'expression écrite aux étudiants de première année à l'Université d'Arizona, mais je ne pense pas que vous iriez jusqu'à dire que c'est ce que la note cherchait à signifier, ni que c'est ce que la note cherchait à nier.

Quoi qu'il en soit, ce qui importe ici, c'est que bien que rien n'ait été réellement caché, il peut échapper au lecteur ordinaire que rien n'a non plus réellement été révélé. Le lecteur non averti présume simplement que les termes utilisés sont les Formes normales, que les caractérisations sont à propos, et qu'on conclurait à la suffisance informationnelle de la contribution si on la recherchait. Le lecteur présume simplement qu'il y a bien là une réponse indirecte qu'il s'agit de décoder.

\section{Cas 4 : Dick Cheney et les tribunaux militaires}

42 La confiance de l'auditoire est trahie de façon similaire par le fonctionnement de certains enthymèmes. Les enthymèmes sont classiquement définis comme des syllogismes incomplets, comportant une prémisse ou une conclusion passée sous silence et reconstituée par l'auditoire (Bitzer 1959). Incontestablement, cette approche rend compte de certains enthymèmes, en particulier de ceux dont c'est la conclusion qui est passée sous silence. Mais on peut concevoir les enthymèmes autrement. Ils peuvent être considérés comme des argumentations dont l'adéquation inférentielle n'a pas été mise en doute. Les enthymèmes sont des argumentations dont on présume que certaines composantes pourraient être explicitées de façon satisfaisante si besoin était (Jackson et Jacobs 1980). ${ }^{15}$

43 Cette présomption revient à une sorte de principe de charité. En général, les locuteurs déploient des argumentations dont les maillons manquants peuvent être produits sur demande, sinon par les locuteurs eux-mêmes, du moins par quelqu'un comme un analyste expert. Et les êtres humains sont à coup sûr dotés d'une imagination réflexive très productive. Il ne fait aucun doute que les locuteurs ordinaires seraient souvent capables d'identifier la prémisse ou la conclusion manquante dans une argumentation, s'il s'avérait nécessaire de la reconstituer et de l'exprimer. Ce qui est moins clair, c'est dans quelle mesure ces locuteurs ou destinataires ont réellement conscience de ces éléments manquants - avant qu'on ne demande à celui qui s'est exprimé de les expliciter.

Considérons une argumentation produite par Dick Cheney alors qu'il était viceprésident, peu de temps après l'attaque du 11 septembre; cette argumentation a été publiée dans un article du New York Times intitulé « Des hauts fonctionnaires prônent le recours à des tribunaux militaires pour les individus soupçonnés de terrorisme " (Buhmiller et Myers 2001).

Des hauts fonctionnaires soutiennent aujourd'hui un ordre présidentiel permettant à des tribunaux militaires de juger des étrangers accusés de terrorisme, au moment où le Pentagone prend des dispositions pour le possible transfert d'immigrants détenus par le Département de la Justice vers des prisons militaires.

L'un d'entre eux a déclaré qu'il était possible que les immigrants détenus aux EtatsUnis par le Département de la Justice en relation avec les événements du 11 septembre soient jugés par un tribunal militaire. Selon ce haut fonctionnaire, ces procès pourraient se tenir en dehors des Etats-Unis, ou même sur des bateaux. 
L'ordre, signé par le Président Bush mardi, confère des pouvoirs considérables au gouvernement, qui peut poursuivre agressivement et dans le plus grand secret des terroristes étrangers sur notre territoire ainsi qu'à l'étranger.

Les fonctionnaires du Département de la Justice ont refusé systématiquement de révéler l'identité des immigrants concernés, ou les charges retenues contre eux. Ils ont déclaré le mois dernier que le nombre total d'individus détenus - y compris ceux qui ont depuis été relâchés - a dépassé le millier, mais ce mois-ci, les responsables ont déclaré qu'ils ne diffuseraient plus de décompte régulier de cet ordre.

«Je n'imaginais pas qu'ils allaient mettre en place ce genre de dispositif pour des individus détenus sur le territoire national ", a affirmé Kevin Ernst, un avocat de Detroit qui représente Farouk Ali-Hamoud, arrêté pour possession de documents d'immigration frauduleux et détenu 25 jours dans la prison du comté de Wayne avant de bénéficier d'un non-lieu le mois dernier. «Ça m'a fichu une sacrée trouille, je peux vous le dire."

Le Vice-Président Dick Cheney a justifié aujourd'hui la mesure prise par Mr Bush, arguant du fait que les terroristes ne sont pas des combattants légitimes, et ne méritent pas la protection que leur offrirait la jurisprudence américaine traditionnelle.

"L'idée au cœur de cette mesure est que quelqu'un qui entre illégalement aux Etats-Unis, qui mène une opération terroriste et tue des milliers d'Américains innocents - des hommes, des femmes, des enfants - n'est pas un combattant légitime ", a déclaré $M$. Cheney.

"Ils ne méritent pas d'être traités comme des prisonniers de guerre », a-t-il ajouté. «Ils ne méritent pas les mêmes garanties et protections que celles dont bénéficierait un citoyen américain à qui serait appliquée la procédure judiciaire normale ».

Le vice-président a assuré l'auditoire que les individus soupçonnés de terrorisme auraient un "procès équitable ", mais a suggéré qu'ils ne méritaient pas un procès qui leur offrirait les mêmes protections que celles qui sont accordées aux citoyens américains. Un tribunal militaire, a-t-il dit, "garantit que ces individus bénéficieront du traitement que nous considérons qu'ils méritent ».

L'argumentation de Cheney vise à soutenir une mesure présidentielle permettant la création de tribunaux militaires secrets pour juger les étrangers accusés de terrorisme. La justification de cette mesure est annoncée dans le premier paragraphe de l'article. Les quatre paragraphes suivants rapportent et expliquent le problème qui se pose à ces tribunaux militaires secrets. Les quatre derniers paragraphes rapportent l'argumentation de Cheney en soutien à ces tribunaux militaires.

En quoi l'argumentation de Cheney consiste-t-elle ici? La position défendue semble assez claire. Il s'agit de récuser une critique implicite adressée aux tribunaux militaires secrets chargés de juger les étrangers accusés de terrorisme. On peut formuler cette récusation ainsi : les étrangers accusés de terrorisme sont des gens qui ne méritent pas les protections judiciaires habituelles. Quel est l'argument avancé en soutien à cette thèse ? Si l'on revient au texte, les choses semblent là encore limpides : les terroristes sont des gens qui ne méritent pas les protections judiciaires habituelles.

Telle est donc l'argumentation : les étrangers accusés de terrorisme sont des gens qui ne méritent pas les protections judiciaires habituelles, parce que les terroristes ne méritent pas les protections judiciaires habituelles.

Il y a là un véritable problème. Comment Cheney peut-il tirer cette conclusion à partir de cet argument? Quelle prémisse manquante peut-on lui attribuer ici? Il existe bien une prémisse qui transformerait l'argumentation en syllogisme valide, mais bien peu de gens la considéreraient comme acceptable. Pour identifier cette prémisse, réduisons 
l'argumentation de Cheney à des relations entre trois catégories : soit A les étrangers accusés de terrorisme, $\mathrm{M}$, les gens qui méritent une protection judiciaire, et $\mathrm{T}$, les terroristes. Appliquons ces conventions au raisonnement de Cheney; on obtient la forme (incomplète) suivante :

Aucun T n'est M

Aucun A n'est M.

Alors, quelle est la prémisse manquante?

On pourrait être enclin à chercher à sauver le syllogisme en proposant une formulation plus prudente de la conclusion: "Certains A ne sont pas M », ou, en d'autres termes, certains étrangers accusés de terrorisme ne méritent pas de protection juridique. Selon cette version, la prémisse manquante serait: "Certains A sont $\mathrm{T}$ » (ou: certains étrangers accusés de terrorisme sont des terroristes).

On peut avec une certaine plausibilité penser que Cheney adhère à cette idée ; mais un problème se pose ici. A présent, la conclusion ne réfute plus la critique adressée aux tribunaux militaires secrets chargés de juger les étrangers accusés de terrorisme. Dire que certains étrangers accusés de terrorisme ne méritent pas de protection judiciaire n'exclut pas la possibilité que d'autres étrangers accusés de terrorisme en méritent une. Et l'objection de la protection judiciaire vise à s'assurer que les innocents ne soient pas traités comme des coupables. Afin de défendre les tribunaux militaires, Cheney doit conclure qu'aucun étranger accusé de terrorisme ne mérite de protection judiciaire.

51 Une seule prémisse manquante peut générer un syllogisme valide; toutes les autres ne sont que des leurres. Cette prémisse doit être que "Tous les A sont T », c'est-à-dire que tous les étrangers accusés de terrorisme sont des terroristes. Seule cette prémisse constitue un lien logique. A présent, le problème posé par l'argumentation de Cheney apparaît clairement.

Il s'agit d'une question de prévention : cette argumentation commet une pétition de principe, et une pétition de principe assez sérieuse ${ }^{16}$. Déterminer qui est et qui n'est pas un terroriste constitue, après tout, l'objectif premier des protections judiciaires. Nous voulons nous assurer que les innocents ne seront pas traités de façon injuste, et pour garantir cela, nous devons traiter ceux qui sont coupables de la même façon, puisqu'il est impossible de déterminer qui est qui, avant que la procédure judiciaire n'arrive à son terme. L'argumentation de Cheney introduit par la bande une prémisse dont la détermination constitue précisément l'enjeu de la procédure judiciaire. Mais les destinataires du discours de Cheney qui n'y regardent pas d'assez près et présument simplement que des prémisses pertinentes et suffisantes pourraient être produites sur demande, pourraient bien laisser passer le fait que l'argumentation ignore ou présuppose précisément ce qui doit être établi.

\section{Conclusion}

L'argumentation est une activité intrinsèquement communicative. Elle implique plus que le simple fait de raisonner, à partir de prémisses, en faveur de conclusions. Argumenter implique d'exprimer et d'interpréter un tel raisonnement. Les assertions, les objections, les réfutations sont réalisées par des actes de communication. Et une 
bonne part de ce qui peut aller de travers dans l'argumentation est liée à la façon dont la communication procède.

Les cas présentés dans cet article montrent que la communication des argumentations dépend de l'application mutuelle de principes pragmatiques, qui constituent un système de standards rationnels. La formulation exacte de ces principes et leur possible systématisation restent des questions ouvertes. Mais je ne vois pas comment expliquer le type de phénomènes que j'ai présentés ici autrement qu'en postulant que de tels principes sont à l'œuvre. Nos intuitions sur les pratiques mauvaises ou fallacieuses peuvent en retour contribuer à spécifier les principes dont nous pensons qu'ils régissent les bonnes argumentations, un peu à l'image du rôle que jouent, dans les théories syntaxiques, les intuitions amenant les locuteurs à juger agrammaticales certaines productions linguistiques.

Ces intuitions mettent au jour une propriété quelque peu paradoxale de nombreux paralogismes basés sur la communication : ils sont rendus possibles par l'exploitation de la présomption même du respect des standards rationnels dont la fonction première est de rendre possible la communication (et la bonne argumentation).

Les locuteurs sont enclins à commettre toutes sortes d'escroqueries ou fraudes verbales. Les pratiques interprétatives qui permettent aux individus de mener à bien l'activité communicative et d'attacher du sens aux messages qui leur sont adressés peuvent aussi les induire en erreur. Mais il est possible d'amener les individus à reconnaître ces mêmes escroqueries et fraudes verbales. Pas toujours, et pas tout le monde. Cependant, les gens savent qu'ils peuvent se faire avoir. Ils savent qu'ils commettent des erreurs. Et ils le savent parce que parfois, ils voient où ça leur est arrivé, ou du moins où ça aurait pu leur arriver. Je suis convaincu que n'importe quel lecteur peut voir où se situe le problème dans au moins certains des quatre cas que j'ai examinés ici.

Mais la question n'est pas simplement que des escroqueries ou fraudes verbales existent. Et ce n'est pas non plus simplement qu'on puisse être amené à les reconnaître lorsqu'elles se produisent. Il faut encore prendre en considération l'attitude qui accompagne cette reconnaissance. Les locuteurs considèrent que ceux qui commettent de tels abus discursifs, ou qui échouent à s'en prémunir (qu'il s'agisse d'eux-mêmes ou de tiers), doivent répondre de ces infractions ou de cette incapacité à s'en défendre. Les principes sur lesquels les locuteurs s'appuient pour se construire une représentation d'une argumentation ou d'un message ont généralement une dimension intrinsèquement normative. Non seulement on s'attend à ce que les locuteurs satisfassent certains standards lorsqu'ils argumentent, mais on attend cela d'eux. Nous utilisons ces principes pragmatiques pour prédire, présumer et guider, mais nous utilisons aussi ces principes pour estimer, évaluer, juger. Cette attitude normative constitue une propriété empirique de l'argumentation, tout autant que la signification $\mathrm{du}$ message qui porte l'argumentation, ou les pratiques communicatives dans le cadre desquelles émerge une argumentation. Et c'est pourquoi les principes de l'argumentation ne sont pas simplement des principes pragmatiques; ce sont des principes pragmatiques normatifs (Jacobs 1999). 


\section{BIBLIOGRAPHY}

Bach, Kent. 1994. « Conversational impliciture », Mind \& Language 9, 124-162

Bitzer, Lloyd F. 1959. « Aristotle’s enthymeme revisited », Quarterly Journal of Speech 45, 399-408

Buhmiller, Elisabeth \& Steven Lee Myers. 2001. « Senior administration officials defend military tribunals for terrorist suspects. A nation challenged : The presidential order », New York Times, 15 novembre, en ligne : http://www.nytimes.com/2001/11/15/us/nation-challenged-presidentialorder-senior-administration-officials-defend.html

Carston, Robyn. 1988. «Implicature, explicature, and truth-theoretic semantics », Ruth Kempson (éd.), Mental representations: The interface between language and reality (Cambridge: Cambridge University Press), 155-181

Chappaquiddick incident. (20 Juillet 2015). Wikipedia. https://en.wikipedia.org/wiki/ Chappaquiddick_incident

Cicourel, Aaron V. 1970. Cognitive sociology (Glencoe, IL : Free Press)

Coleman, Linda \& Paul Kay. 1981. « Prototype semantics : The English word lie », Language 57, 26-44

Damore, Leo. 1988. Senatorial privilege: The Chappaquiddick cover-up (Washington, D. C. : Regnery Publishing)

Eemeren, Frans H. van \& Rob Grootendorst. 1984. Speech acts in argumentative discussions (Dordrecht : Foris)

Eemeren, Frans H. van \& Rob Grootendorst. 1992. Argumentation, communication, and fallacies : A Pragma-Dialectical perspective (Hillsdale, NJ : Erlbaum)

Gauker, Christopher. 1986. "The principle of charity ", Synthese 69, 1-25

Grice, H. Paul. 1989. « Logic and conversation », Studies in the way of words (Cambridge : Harvard University Press), 22-40. Traduction française : « Logique et conversation », Communication 30 (1979), 57-72

Horn, Laurence. 1984. « Toward a new taxonomy for pragmatic inference : Q-based and R-based implicature ", Deborah Schiffrin (éd.), Meaning, form, and use in context : Linguistic applications. GURT '84 (Washington : Georgetown University Press), 11-42

Jackson, Sally \& Scott Jacobs. 1980. « Structure of conversational argument : Pragmatic bases for the enthymeme », Quarterly Journal of Speech 66, 251-265

Jacobs, Scott. 1993. «The problem of indirect communication for descriptive and normative models of argumentation : Teddy Kennedy drives off the bridge at Chappaquiddick ", Raymie E. McKerrow (éd.), Argument and the postmodern challenge (Annandale, VA : Speech Communication Association), 194-199

Jacobs, Scott. 1999. « Argumentation as normative pragmatics », Frans H. van Eemeren, Rob Grootendorst, J. Anthony Blair \& Charles A. Willard (éds.), Proceedings of the fourth ISSA conference on argumentation. (Amsterdam : SICSAT), 397-403

Jacobs, Scott. 2002. «Language and interpersonal communication », Mark L. Knapp \& John A. Daly (eds.), Handbook of interpersonal communication ( $3^{\mathrm{e}}$ éd.) (Thousand Oaks, CA : Sage Publications), 213-239 
Johnson, Ralph Henry \& J. Anthony Blair. 2006. Logical self-defense (New York : International Debate Education Association)

Kennedy's television statement to the People of Massachusetts. 26 juillet 1969. New York Times, p. 10, cols. 3-8.

Levinson, Stephen C. 2000. Presumptive meanings: The theory of generalized conversational implicature (Cambridge, MA : MIT Press).

Polcar, Leah \& Scott Jacobs. 1998. « Evasive answers : Reframing multiple argumentative demands in political interviews ", James F. Klumpp (éd.), Argument in a time of change : Definitions, frameworks, and critiques. Proceedings of the tenth NCA/AFA conference on argumentation (Annandale, VA : National Communication Association), 226-231

Sacks, Harvey. 1972. « On the analyzability of stories by children », John J. Gumperz \& Dell Hymes (éds.), Directions in sociolinguistics : The ethnography of communication (New York, NY : Rinehart \& Winston), 325-345

Simon, Roger. 1990. «How a murderer and rapist became the Bush campaign's most valuable player », Baltimore Sun, 11 novembre, en ligne : http://articles.baltimoresun.com/1990-11-11/ features/1990315149_1_willie-horton-fournier-michael-dukakis

Sperber, Dan \& Deirdre Wilson. 1995. Relevance : Communication and cognition ( $2^{\mathrm{e}}$ éd.) (Oxford : Blackwell)

Ware, B. Lee \& Wil A. Linkugel. 1973. «They spoke in defense of themselves : On the generic criticism of apologia », Quarterly Journal of Speech 59, 273-283

Wilson, Neil L. (1959). « Substances without substrata », The Review of Metaphysics 12, 521-539

Zipf, George Kingsley. 1949. Human behavior and the principle of least effort (Cambridge : AddisonWesley)

\section{NOTES}

1. Nous avons conservé le terme "implicature » pour traduire l'anglais implicature au motif que c'est la solution la plus communément admise aujourd'hui parmi les commentateurs de Grice ; en cela, nous n'avons pas suivi la proposition du traducteur de son article séminal de 1979, qui suggérait d'utiliser « implicitation », suggestion qui n'a généralement pas été reprise. N.d.T.

2. Je remercie Michael van Lambalgan pour cette heureuse formulation.

3. Principe de coopération: Que votre contribution conversationnelle corresponde à ce qui est exigé de vous, au stade atteint par celle-ci, par le but ou la direction acceptés de l'échange parlé dans lequel vous êtes engagé.

Maximes conversationnelles :

QUANTITÉ :

Que votre contribution contienne autant d'information qu'il est requis (pour les visées conjoncturelles de l'échange).

Que votre contribution ne contienne pas plus d'information qu'il n'est requis.

QUALITÉ : Que votre contribution soit véridique :

N'affirmez pas ce que vous croyez être faux.

N'affirmez pas ce pour quoi vous manquez de preuves.

RELATION : Parlez à propos.

MODALITÉ : Soyez clair.

Évitez de vous exprimer avec obscurité. 
Évitez d'être ambigu.

Soyez bref (ne soyez pas plus prolixe qu'il n'est nécessaire).

Soyez méthodique (Grice 1979 : 61-62).

4. Le stimulus ostensif est suffisamment pertinent pour mériter d'être traité par le destinataire.

Le stimulus ostensif est le stimulus le plus pertinent qui soit compatible avec les aptitudes et préférences du communiquant.

5. Le Principe-Q (centré sur l'économie des efforts de l'allocutaire) :

Que votre contribution soit suffisante

Dites autant que vous le pouvez (étant donné $\mathrm{R}$ )

Le Principe-R (centré sur l'économie des efforts du locuteur) :

Que votre contribution soit nécessaire

$\mathrm{Ne}$ dites pas plus que ce qui est requis (étant donné $\mathrm{Q}$ ).

6. Principe- $Q$ : Ce qui n'est pas dit, n'est pas le cas (Ce que vous ne dites pas est faux)

Maxime du locuteur. N'affirmez rien qui soit informationnellement plus faible que ce que votre connaissance du monde vous permet d'affirmer.

Corollaire du récepteur. Considérez que le locuteur a produit l'affirmation la plus forte possible qui soit cohérente avec ce qu'il sait.

Principe-M : Ce qui est dit d'une façon anormale n'est pas normal.

Maxime du locuteur. Indiquez qu'une situation est anormale, non-stéréotypique, en utilisant des expressions marquées qui contrastent avec celles que vous utiliseriez pour décrire les situations normales, stéréotypiques, correspondantes.

Corollaire du récepteur. Ce qui est dit d'une façon anormale indique une situation anormale.

Principe-I : Ce qui est exprimé simplement est exemplifié de façon stéréotypique.

Maxime du locuteur. Produisez l'information linguistique minimale qui soit suffisante pour atteindre vos objectifs communicationnels.

Corollaire du récepteur. Amplifiez le contenu informationnel de l'énoncé produit par le locuteur en trouvant l'interprétation la plus spécifique qui soit cohérente avec le savoir relevant du sens commun, jusqu'à ce que vous identifiiez ce qui vous semblait être visé par le locuteur.

7. On peut distinguer les implicatures conversationnelles des explicatures conversationnelles (Carston 1988), ou des implicitures conversationnelles (Bach 1994); toutes reposent sur des principes similaires.

8. Les enquêtes relatant les faits sont légion. On trouvera sur le site en anglais de Wikipedia une synthèse lisible du scandale : Chappaquiddick incident, 2015 (l'article correspondant sur le site de Wikipédia en français est excessivement succinct ; n.d.t.). Voir Damore (1988) pour une recension détaillée.

9. La présomption interprétative des Formes normales peut être vue comme une sorte de corollaire à l'interaction entre les principes M et I de Levinson (2000) (voir note 6). Le principe M pose qu'on attend des locuteurs qu'ils signalent les situations anormales ou non stéréotypiques. Le principe I pose qu'on attend des destinataires qu'ils reconstruisent les informations spécifiques issues du savoir commun jusqu'à ce qu'ils estiment avoir trouvé ce que le locuteur cherchait à exprimer. Cette sorte d'activation du savoir commun en fonction d'un objectif déterminé est mise en branle en l'absence d'indication, de la part du locuteur (par le Principe M), que ce ne doit pas être le cas.

10. La formulation du principe d'A-propos informationnel vise à éclairer pourquoi la supermaxime de Qualité de Grice réfère à la « contribution » du locuteur plutôt qu'à ce que le locuteur « dit » ou "affirme » (comme dans les sous-maximes de Qualité). Ce principe a également une affinité évidente avec la théorie de la pertinence de Sperber et Wilson, même si "pertinence ", dans notre formulation, renvoie à la façon dont l'information est utile pour la réalisation des buts présumés de l'échange. 
11. Il n'y a aucune raison de supposer que Kennedy ou son assistant aient été au courant de ce fait. Et Kennedy reconnait ailleurs dans son discours que d'autres hommes et «filles" participaient à la fête.

12. Ainsi que l'a dit Kennedy au début de son discours : "Avant d'être entendu par la Cour, il aurait été inapproprié de ma part de commenter ces événements. Mais ce soir, je suis libre de vous dire ce qui s'est passé et de vous dire ce que ça signifie pour moi. »

13. Il peut être également éclairant d'y voir une sorte de problème de coordination, une incapacité à produire et traiter des assertions et arguments qui soient mutuellement intelligibles (cf. van Eemeren et Grootendorst 1992).

14. Ou encore, on pourrait l'interpréter comme « la seule fois, parmi les nombreux exemples de prisonniers condamnés pour meurtre et ayant bénéficié d'une autorisation de sortie pour le week-end, où le prisonnier en question a commis un crime haineux, comme de sauvagement violer et battre une femme alors que son fiancé en était réduit à écouter ses cris, impuissant ». Cette interprétation se rapproche de la première, et diffère de la seconde.

15. Pour une analyse similaire, voir la perspective gricéenne proposée au chapitre 6 de van Eemeren et Grootendorst (1984), « Prémisses non exprimées dans une discussion rationnelle ». Ils traitent les prémisses non exprimées comme des engagements implicités qui doivent pouvoir être restitués sur demande.

16. Bien sûr, comme toute implicature conversationnelle, cette interprétation peut être mise à mal par un cadrage adapté. Que cette argumentation ait été sortie de son contexte ou qu'il s'agisse bien de l'argumentation défendue par Cheney, c'est en tout cas l'argumentation que l'article du Times prête à Cheney.

\section{ABSTRACTS}

Many of the kinds of messages that argumentation scholars call fallacies involve something false or mistaken, but something less than full-fledged lies or less even than just the overt and explicit expression of premises or reasons with false propositional content. How such verbal frauds work is the subject of this paper. Based on the analysis of four such deceiving messages, it shows the importance of pragmatic principles in understanding how arguments work and how they go awry. It suggests that verbal frauds are made possible by exploiting the very presumption of rational standards that make communication (and good argument) possible in the first place. The principles by which people reason to representations of arguments and messages in general have an intrinsically normative quality. That normative attitude is as much an empirical property of argumentation as is the meaning of the message that conveys an argument or the occurrence of the practices that produce an argument.

Bon nombre de messages que les spécialistes en argumentation considèrent comme des paralogismes ont bien quelque chose de faux ou d'erroné, mais ne reposent pas pour autant sur des mensonges caractérisés. Cet article cherche à élucider le fonctionnement de telles fraudes verbales. A partir de l'analyse de quatre exemples de tels messages trompeurs, il met en évidence l'importance des principes pragmatiques dans la compréhension des mécanismes argumentatifs, et de la façon dont ils peuvent être dévoyés. Il montre que les pratiques interprétatives qui permettent aux individus de mener à bien l'activité communicative et d'attacher du sens aux messages qui leur sont adressés peuvent aussi les induire en erreur. Les principes sur lesquels les 
locuteurs s'appuient pour se construire une représentation d'une argumentation ou d'un message ont généralement une dimension intrinsèquement normative. Cette attitude normative constitue une propriété empirique de l'argumentation, tout autant que la signification du message qui porte l'argumentation, ou les pratiques communicatives dans le cadre desquelles émerge une argumentation.

\section{INDEX}

Keywords: informational aptness, informational sufficiency, interpretative pragmatic principles, normal forms, normative pragmatics

Mots-clés: à propos informationnel, formes normales, pragmatique normative, principes pragmatiques d'interprétation, suffisance informationnelle

\section{AUTHORS}

\section{SCOTT JACOBS}

Université d'Illinois 